\title{
siRNA-mediated knockdown of VEGF-A, VEGF-C and VEGFR-3 suppresses the growth and metastasis of mouse bladder carcinoma in vivo
}

\author{
FENG WANG, HUI-MING LI, HUI-PING WANG, JIA-LIE MA, XIA-FANG CHEN, \\ FANG WEI, MIAO-YING YI and QIAN HUANG
}

Experimental Center, First People's Hospital, Medical School, Shanghai Jiaotong University, Shanghai 200080, P.R. China

Received April 9, 2010; Accepted June 11, 2010

DOI: $10.3892 /$ etm.2010.113

\begin{abstract}
The growth of solid tumors is highly dependent on the formation of new blood and/or lymph vessels. Furthermore, metastases often disperse via newly formed blood or lymphatic vessels within the tumor, particularly in the case of epithelium-derived tumors. Since vascular endothelial growth factor (VEGF) signaling plays a vital role in angiogenesis and lymphangiogenesis, we used the small interfering RNA (siRNA) approach to selectively down-regulate VEGF-A, VEGF-C or VEGF receptor 3 (VEGFR-3) expression in bladder transitional carcinoma cells derived from T739 mice in an attempt to suppress tumor growth and metastasis in vivo. The synthetic siRNA was introduced into the tumor tissues by in vivo electroporation. The knockdown of VEGF-A, VEGF-C and VEGFR-3 expression significantly delayed tumor growth and reduced tumor metastasis compared to the negative controls. Thus, electroporation-mediated siRNA delivery to block the VEGF signaling pathway may provide a novel approach for the treatment or prevention of solid tumor growth and metastasis.
\end{abstract}

\section{Introduction}

Tumor growth and metastasis are angiogenesis- and lymphangiogenesis-dependent events (1). By inducing endothelial cell proliferation and migration, vascular endothelial growth factor (VEGF) signaling plays an important role in promoting tumor metastasis, and this pathway has become a promising target for cancer therapy (2). The secreted glycoprotein VEGF-A causes proliferation, sprouting, migration and tube formation of endothelial cells, and has been demonstrated to play a crucial role in tumor angiogenesis (3). VEGF-A binds

Correspondence to: Professor Qian Huang, Experimental Center, First People's Hospital, Medical School, Shanghai Jiaotong University, Shanghai 200080, P.R. China

E-mail: qhuang@sjtu.edu.cn

Key words: bladder carcinoma, small interfering RNA, vascular endothelial growth factor (VEGF)-A, VEGF-C, VEGF receptor 3 to the transmembrane receptor VEGFR-2 $(4,5)$, leading to the activation of the downstream signal transduction pathway and endothelial proliferation-related genes (6). Increased VEGF-A expression levels have been detected in numerous types of human cancer, including lung, gastrointestinal tract, kidney, thyroid, bladder, ovarian and cervical tumors (7). Animal models have confirmed that the growth of a large variety of tumors is suppressed by the inhibition of VEGF-A signaling. For example, our previous study showed that blocking VEGF-A expression effectively inhibited bladder cancer angiogenesis, as well as tumor growth and metastasis (8).

By enabling cancer cells to gain access to the lymphatic system, lymph vessels in tumors provide another route for metastasis. It was recently discovered that VEGF-A induces lymphatic vessel growth in tumors and promotes metastasis to local lymph nodes (5). VEGF-C and its receptor VEGFR-3 also stimulate the proliferation and migration of lymphatic endothelial cells, which in turn enhances the assembly of new lymphatic vessels $(9,10)$. The binding of VEGF-C to VEGFR-3 stimulates lymphatic growth in tumors (10), and patients with high intratumoral levels of VEGF-C have poorer prognoses than those who express little or no VEGF-C (11). Recent studies have shown that overexpression of VEGF-C in several types of human tumors, including bladder cancer, may greatly increase intratumoral lymphangiogenesis, leading to significant increases in regional lymph node metastasis $(4,5)$. These findings indicate that blocking both tumor angiogenesis and lymphangiogenesis concurrently may provide an optimal strategy for cancer therapy.

Small interfering RNAs (siRNAs) inhibit the expression of specific target genes via the evolutionarily conserved mechanism of RNA interference (RNAi) $(6,12)$. Transgenic RNAi is a powerful tool for silencing mammalian genes with a high degree of specificity and efficacy, and is considered a promising therapeutic approach for treating various diseases, including cancer $(13,14)$. In this study, we transfected siRNA constructs to knock down the expression of VEGF-A, VEGF-C and VEGFR-3 in green fluorescent protein (GFP)-expressing T739 mouse bladder transitional carcinoma (BTT) cells, and investigated the effects on tumor growth and metastasis. siRNA-mediated knockdown of VEGF signaling was found to suppress tumor growth and reduce tumor metastasis in vivo. 


\section{Materials and methods}

siRNA synthesis. siRNA sequences were designed using the software available at http://www.ambion.com/techlib/misc/ siRNA_design.html followed by a BLAST search to eliminate non-unique targeting sequences. Three sequences targeting VEGF-A, four targeting VEGF-C and six targeting VEGFR-3 were selected.

VEGF-A siRNA sequences containing BamHI/HindIII sites were synthesized and cloned into pSilencer puro $\mathrm{H} 1$ vector according to the manufacturer's protocol (Ambion). After transformation, the presence and orientation of the VEGF-A siRNA inserts in plasmid DNA clones were confirmed by double digestion with BamHI and HindIII enzymes (Takara). The plasmid DNA (isolated with mini-prep kits; Qiagen) was also sequenced to confirm the presence of the VEGF-A siRNAs. VEGF-C and VEGFR-3 siRNAs were synthesized in vitro and purified using Silencer ${ }^{\circledR}$ siRNA construction kits (Ambion) according to the product manuals. The end product was a double-stranded 21-mer siRNA with 3 ' terminal uridine dimers that effectively reduce the expression of target mRNA when transfected into mammalian cells. The scrambled siRNA negative control had the same construct-containing sequence and no homology with any genes. siRNAs used in vivo were synthesized and modified by Shanghai GenePharma Co., Ltd.

Cell cultures and transfections. BTT-T739-GFP mouse bladder cancer cells stably expressing GFP were grown in RPMI-1640 medium (Invitrogen) containing 10\% FBS with penicillin/streptomycin antibiotics (100 units of penicillin and $100 \mu \mathrm{g}$ of streptomycin per ml). VEGF-A siRNA plasmid DNA, VEGF-C and VEGFR-3 siRNAs were transfected using Lipofectamine 2000 (Invitrogen). Cells $\left(8 \times 10^{4}\right)$ were seeded in 24-well plates the day before transfection. Plasmid DNA $(1 \mu \mathrm{g})$ or siRNA and $(2 \mu \mathrm{l})$ Lipofectamine 2000 were applied according to the protocol provided by the manufacturer. Each condition was performed in triplicate. Cells transfected with a scrambled siRNA construct were used as the negative control. Total RNA was isolated from cells using TRIzol reagent (Invitrogen) $48 \mathrm{~h}$ after transfection.

Real-time RT-PCR analysis. cDNA was amplified from total RNA using a reverse transcription system (Promega). SYBR Green quantitative PCR amplifications were performed in a MJ Opticon2 System (BioRad). Reactions were carried out in 20- $\mu 1$ volumes containing $10 \mu \mathrm{l}$ of $2 \mathrm{X}$ SYBR Green PCR MasterMix (Takara). The cycle profile for real-time PCR was $95^{\circ} \mathrm{C}$ for $2 \mathrm{~min}$ followed by 40 cycles of $95^{\circ} \mathrm{C}$ for $30 \mathrm{sec}, 58^{\circ} \mathrm{C}$ for $30 \mathrm{sec}$ and $72^{\circ} \mathrm{C}$ for $30 \mathrm{sec}$. Glyceraldehyde 3-phosphate dehydrogenase (GAPDH) was used as the internal control to normalize gene expression levels. Ct values were collected, and the average $\Delta \mathrm{Ct}$ for each group was calculated using the following formula: $\Delta \mathrm{Ct}=$ average target gene $\mathrm{Ct}$ - average GAPDH Ct. $\Delta \Delta \mathrm{Ct}$ was calculated by the formula: $\Delta \Delta \mathrm{Ct}=$ $\Delta \mathrm{Ct}$ of control group - $\Delta \mathrm{Ct}$ of the siRNA-treated group. The relative expression levels of target genes were calculated using the $2^{-\Delta \Delta \mathrm{Ct}}$ method. Each reaction was performed in triplicate.

ELISA and Western blotting. To measure VEGF-A concentration in the supernatant from VEGF-A siRNA plasmid-transfected BTT-T739-GFP cells, a mouse VEGF-A Quantikine Colorimetric Sandwich ELISA kit (R\&D Systems) was used. All tests were performed in triplicates. The determination of VEGF-C protein expression in VEGF-C siRNA-transfected BTT-T739-GFP cells was carried out using Western blotting according to the standard method.

In vivo siRNA therapy. Animals in this research were maintained and studied according to local and national regulations on animal welfare, and the licensing committee approved the experiments. Twenty-two male 6- to 8-week-old T-739 mice were subcutaneously injected with $1 \times 10^{6}$ tumor cells/mouse. A week later, when the tumors were palpable, the mice were randomly assigned into each of three groups and anesthetized by an intraperitoneal injection of $50 \mathrm{mg} / \mathrm{kg}$ pentobarbital. Each mouse received an intratumoral injection of $10 \mu \mathrm{g}$ siRNA/mouse: scrambled siRNA (negative control group, $\mathrm{NC} ; \mathrm{n}=6)$, siVEGF-C + siVEGFR-3 $(\mathrm{n}=8)$ or siVEGF-A $(\mathrm{n}=8)$. Following the injections, the tumors were electroporated with 8 pulses of $900 \mathrm{~V} / \mathrm{cm}$ for $100 \mu \mathrm{m}$ at $1 \mathrm{~Hz}(15)$. Injections and electroporations were repeated once every three days a total of 5 times. Tumor length and width were measured with calipers, and tumor volume was calculated with the formula: Volume = $0.52 \times$ (width $\mathrm{x}$ length ${ }^{2}$ ). Animals were sacrificed 1 week after the final injection, and livers, lungs and tumors were excised for molecular biology and histological analysis. The regional lymph nodes from axillary and femoral regions were isolated, and lymph node volume was calculated as: Volume $=0.5 \mathrm{x}$ (width $\mathrm{x}$ length $\mathrm{x}$ height).

Relevant gene expression level in tumor tissues. VEGF-A, VEGF-C, VEGFR-2 and VEGFR-3 mRNA expression levels in tumor tissues were detected to ascertain whether the siRNAs effectively knock down the relevant gene expression in vivo. The expression level of these genes also reflects the state of angiogenesis and lymphogenesis in tumors. To accomplish this, total RNA was extracted from tumor tissues after siRNA treatment, and cDNA and real-time PCR was carried out according to the the method described above. Each realtime PCR reaction was carried out in triplicates.

Analysis of tumor metastasis. GFP-positive tumor cells in unfixed whole organ samples were visualized using fluorescent microscopy. Livers and lungs were then fixed in neutral formalin for histological analysis.

Visualization of lymph nodes by Carnalym lymphatic tracer. To visualize the regional lymph nodes, $30 \mu 1$ of $50 \mathrm{mg} /$ $\mathrm{ml}$ Carnalym lymphatic tracer (provided by the Chengdu Institute of High-Technology Medicine) was injected into the tumors. Twenty-four hours later, the animals were sacrificed, and the regional lymph nodes from the axillary and femoral regions were isolated for observation under a stereomicroscope.

Statistical analysis. Statistical analyses were carried out using SPSS software (version 11.0). Data were expressed as the means \pm SEM and analyzed by one-way ANOVA, the independent-samples t-test and the least significant difference test. $\mathrm{p}<0.05$ was considered significant. 
A

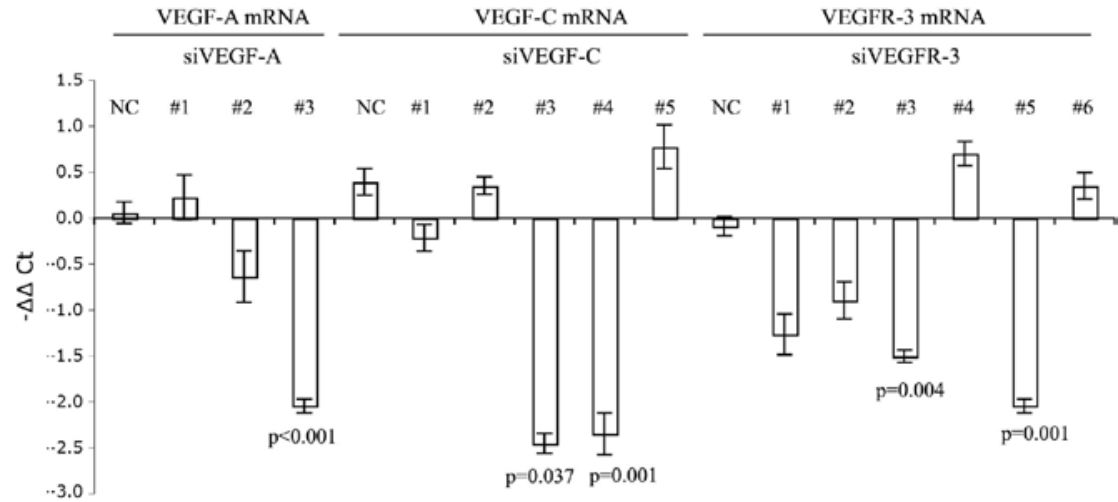

B

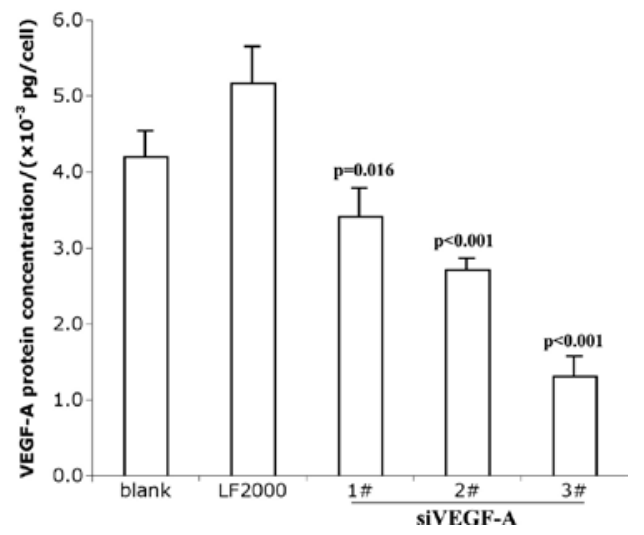

C

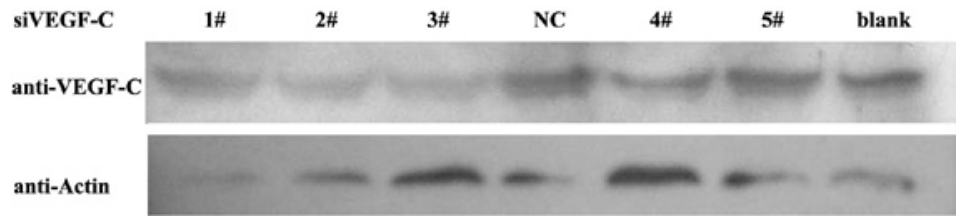

Figure 1. Real-time RT-PCR (A), ELISA (B) and Western blotting (C) showing relative VEGF-A, VEGF-C and VEGFR-3 mRNA and protein levels in BTTT739 cells transfected with various siRNA constructs. Compared to untransfected control levels, significant decreases in target gene expression were achieved with the following siRNA constructs: siVEGF-A \#3, siVEGF-C \#3 and 4, siVEGFR-3 \#2 and 5. A negative control siRNA (NC; scrambled sequence) did not significantly affect the expression of VEGF-A, VEGF-C or VEGFR-3. Data represent the means \pm SEM from 6 (A) or 3 (B) independent experiments. A p-value represents significance vs. the blank control.

\section{Results}

siRNA-mediated silencing of VEGF-A, VEGF-C and VEGFR-3 in mouse bladder cancer cells. The inhibitory effect of siRNAs targeting the VEGF pathway was tested in BTT-T739-GFP cells, a mouse bladder cancer cell line that expresses high levels of VEGF-A, VEGF-C and VEGFR-3 (16). VEGF-A expression was measured by real-time RT-PCR following in vitro transfection with one of three plasmids encoding unique VEGF-A siRNA sequences (siVEGF-A \#1-3). Of the three, siVEGF-A \#3 greatly reduced VEGF-A expression relative to the negative control. Specific siRNAs against VEGF-C (siVEGF-C \#3 and 4) and VEGFR-3 (siVEGFR-3 $\# 3$ and 5) also significantly reduced target gene expression relative to the negative controls $(\mathrm{p}<0.05$; Fig. 1A). VEGF-A protein levels in the cell culture supernatant were measured by ELISA, and siVEGF-A \#3 showed the lowest level (Fig. 1B). siVEGF-C \#3 showed the most inhibited level as noted by Western blotting (Fig. 1C). Thus, the most effective siRNA sequences, siVEGF-A \#3 (ATGTGAATGCAGACCAAAG), SiVEGF-C \#3 (AACAGAGGCCCAAGTCTGTGT) and siVEGFR-3 \#5 (GAAGCCCAATCAATAACTG) were used for subsequent in vivo experiments.

VEGF-A, VEGF-C and VEGFR-3 siRNAs inhibit the growth of BTT-T739-GFP cell xenografts. To determine whether our siRNAs inhibited tumor growth in vivo, BTT-T739-GFP cells were subcutaneously injected into adult mice $\left(1 \times 10^{6}\right.$ cells/ mouse). Tumors were allowed to develop for 1 week and then a regimen of siRNA therapy was administered. siRNAs were delivered directly into the tumor by electroporation once every 3 days a total of 5 times, and tumor growth was monitored over 26 days ( 7 days prior to and during, and 7 days following treatment). Tumor volume was calculated by measuring their width and length. As shown in Fig. 2, delivering siVEGF-A alone or siVEGF-C + siVEGFR-3 dramatically reduced the growth rate of BTT-T739 tumors relative to the scramble siRNA-transfected negative controls. Significant differences in tumor size were noted on Day 19, and the margin of difference widened over the remaining 7 observation days. By the end of the 26-day period, the mean tumor size in the siVEGF-A group was $36.1 \%$ smaller than that in the negative control group. Delivering siVEGFR-3 together with siVEGF-C had an effect similar to that of siVEGF-A alone, exhibiting significant effects by Day 19 and reducing tumor growth by $55.4 \%$ by the end of the 26-day observation period.

mRNA expression of related genes was down-regulated in tumor tissues after siRNA therapy. Real-time RT-PCR was applied to measure the mRNA levels of VEGF-A, VEGFR-C, VEGFR-2 and VEGFR-3 in tumor tissues after siRNA therapy. VEGF-A was significantly reduced in the siVEGF-A group, which showed that siVEGF-A efficiently knocked down the expression of VEGF-A in the tumors. VEGF-A receptor, VEGFR-2, which partially represents vascular vessel density, was also down-regulated in both siRNA therapy groups 


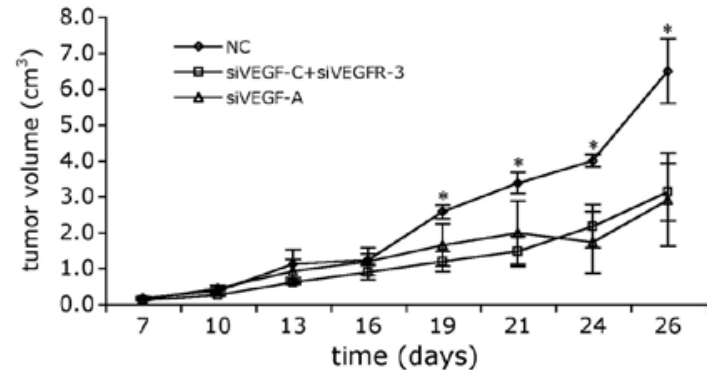

Figure 2. Tumor growth in bladder tumor-bearing mice treated with VEGF siRNA. Mice were subcutaneously injected with BTT-T739-GFP cells and, beginning 7 days later, tumors were injected with VEGF-targeting siRNAs every 3 days a total of 5 times. Compared to the negative controls (NC), tumor volumes were significantly reduced in both VEGF siRNA-treated groups relative to the NC group (injected with scrambled siRNA) beginning at Day 19, and the margin of difference increased up to Day 26. Data points represent the means $\pm \mathrm{SEM}$; ${ }^{*} \mathrm{p}<0.05$ (NC vs. siVEGF-A or siVEGF-C + siVEGFR-3).

(Fig. 3). VEGF-C and VEGFR-3 expression was decreased in the siRNA therapy groups, but without significance. The results showed that angiogenesis and lymphangiogenesis in the tumors were inhibited after siRNA therapy.

Effects of siRNA therapy on lymph node volume. Lymph nodes play important roles during tumor metastasis. Groin and axilla lymph nodes ipsilateral and contralateral to the tumor were dissected and measured after the 26-day observation period. The volume of the lymph nodes was calculated as Volume $=$ $0.52 \mathrm{x}$ (length $\mathrm{x}$ width $)^{2}$. The volume of the ipsilateral groin lymph nodes was much larger in both treatment groups than in the controls (Fig. 4), likely due to the reactive proliferation of the lymph nodes (22). On the other hand, the volume of the contralateral groin lymph nodes and axilla lymph nodes was not affected in either siRNA treatment group.

Reduced metastases following treatment with siVEGF-A or siVEGF-C + siVEGFR-3. BTT-T739-GFP cells express GFP; thus, tumor metastasis is directly observed in whole organs under a fluorescent microscope. Whole lungs and livers were removed 26 days after tumor cell injection, and the numbers of BTT-T739-GFP aggregates were counted. In the siVEGF-C + siVEGFR-3 group, no obvious reduction in lung metastasis and reduced liver metastasis were observed compared to the negative control group. In the group treated with siVEGF-A alone, metastases in the lung were reduced compared to the

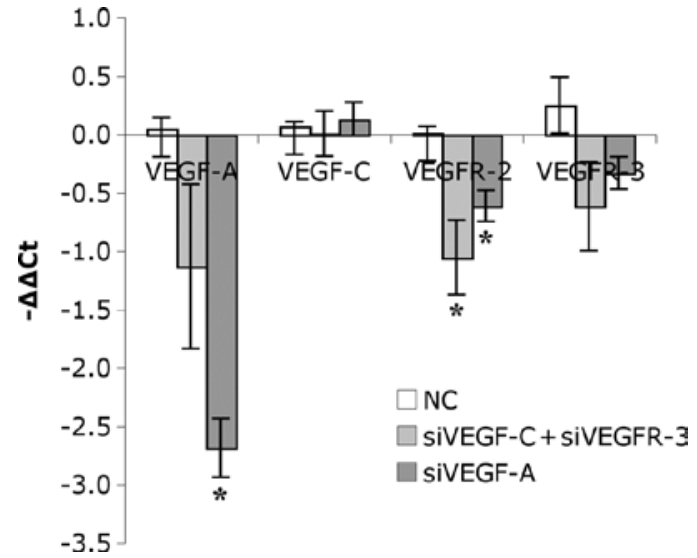

Figure 3. Real-time RT-PCR indicating VEGF-A, VEGF-C, VEGFR-2 and VEGFR-3 mRNA levels after siRNA therapy. Total RNA was extracted from tumors after siRNA therapy, and real-time RT-PCR was applied. VEGF-A and its receptor VEGFR-2 were significantly down-regulated in the siVEGF-A group compared to the negative control (NC). VEGF-C and VEGFR-3 expression was knocked down in the siRNA therapy groups without significant difference. ${ }^{*} \mathrm{p}<0.05$ vs. NC.

negative control group, and no metastatic tumor cells were found in the livers (Table I).

Carnalym shows lymphatic drain from the tumors. Regional lymph nodes were dissected and observed $24 \mathrm{~h}$ after the injection of Carnalym into the tumors. Blackened regional lymph nodes or lymphatics were noted in the axillary and femoral regions (Fig. 5) suggesting drainage from the tumors.

\section{Discussion}

Previous studies have demonstrated that VEGF-C-targeting RNAi effectively suppresses the lymphatic metastasis of human breast cancer cells (17) and that, by inhibiting angiogenesis, VEGF-targeted RNAi suppresses retinoblastoma and Ewing's sarcoma $(18,19)$. In this study, we designed and selected siRNAs targeting VEGF-A, VEGF-C and VEGFR-3, which effectively reduced target gene expression in bladder cancer cells in vitro. These siRNAs were then tested for their effects on bladder cancer cell proliferation and metastasis in vivo. Our results showed that tumor growth was significantly suppressed after treating tumors with siVEGF-A as well as siVEGF-C + siVEGFR-3 relative to the negative controltreated tumors. VEGF-A, VEGF-C, VEGFR-2 and VEGFR-3 expression was also reduced in the tumors after treatment,

Table I. Grade of tumor metastasis in the liver and lung.

\begin{tabular}{|c|c|c|c|c|c|c|c|c|c|}
\hline & \multirow[b]{2}{*}{$\mathrm{n}$} & \multicolumn{4}{|c|}{ Lung (\%) } & \multicolumn{4}{|c|}{ Liver $(\%)$} \\
\hline & & + & ++ & +++ & ND & + & ++ & +++ & ND \\
\hline Negative control & 6 & 33.33 & 16.67 & 50.00 & 0.00 & 33.33 & 16.67 & 0.00 & 50.00 \\
\hline siVEGF-C + siVEGFR-3 & 8 & 37.50 & 12.50 & 50.00 & 0.00 & 25.00 & 0.00 & 0.00 & 75.00 \\
\hline siVEGF-A & 8 & 25.00 & 37.50 & 0.00 & 37.50 & 0.00 & 0.00 & 0.00 & 100.00 \\
\hline
\end{tabular}

ND, not detected. 
A
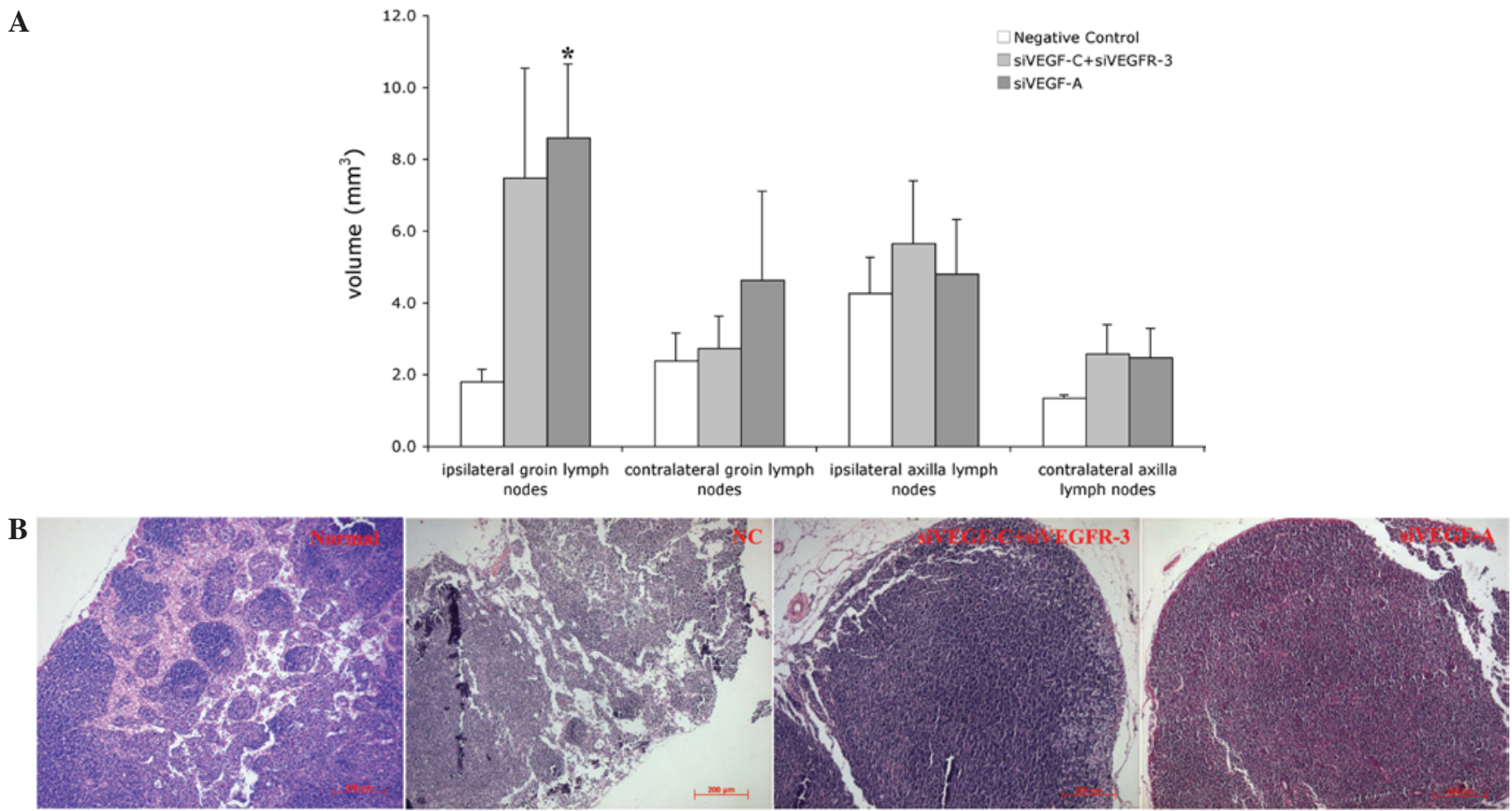

Figure 4. Regional lymph nodes after treatment with siRNAs. (A) Lymph node volumes. Ipsilateral groin lymph nodes were significantly larger in mice treated with siVEGF-A and siVEGF-C + siVEGFR-3 relative to the negative control (NC; scramble siRNA-injected mice). Data represent the means \pm SEM. * $\mathrm{p}=0.043$ for siVEGF-A $(n=8)$ vs. NC and $\mathrm{p}=0.158$ for siVEGF-C + siVEGFR-3 $(n=8)$ vs. NC $(n=6)$. (B) Representative sections of normal and groin lymph nodes ipsilateral to the tumor removed 26 days after tumor cell injection. Normal lymph nodes (left) and tumors were treated with scrambled siRNA (second from left), siVEGF-C + siVEGFR-3 (third from left), or siVEGF-A (right), as described in Fig. 2. An apparent reactive proliferation occurred in the nodes of the VEGF siRNA-treated mice.
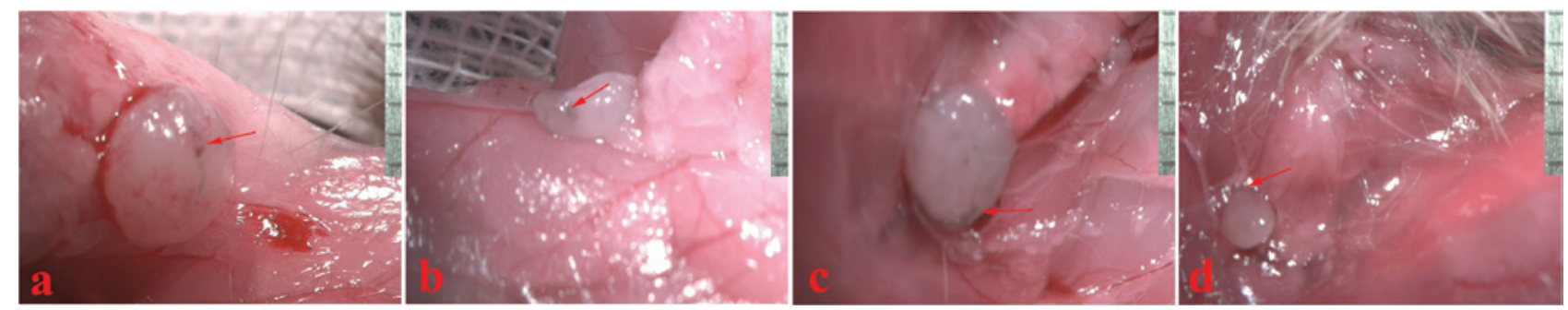

Figure 5. Visualization of lymph nodes after Carnalym lymphatic tracer injection into tumors. Carnalym (30 $\mu \mathrm{lof} 50 \mathrm{mg} / \mathrm{ml})$ was injected into tumors $24 \mathrm{~h}$ before the animals were sacrificed. The lymph nodes were isolated and observed under a stereomicroscope. The arrows show the blackened lymphatics in the ipsilateral groin lymph node (a), contralateral groin lymph node (b), ipsilateral axilla lymph node (c) and contralateral axilla lymph node (d) of the same mouse (x60). The scale on the right indicates $1 \mathrm{~mm}$.

showing that the siRNAs used efficiently interfered with the relevant gene expression both in vitro and in vivo and led to reduced angiogenesis and lymphangiogenesis in the tumors. In addition, siVEGF-A therapy significantly reduced the incidence of lung and liver metastases, and a combinational therapy of siVEGF-C + siVEGFR-3 significantly reduced liver metastasis.

Notably, the injection of VEGF-A siRNAs into BTT-T739 tumors resulted in the enlarged volume of ipsilateral groin lymph nodes in comparison to the controls, which was morphologically confirmed to contain a reactive proliferation. These regional lymph nodes showed drainage from the tumors, which was visualized by Carnalym lymphatic tracer. Another research group also reported that lymphatic drainage near the tumor sites enlarged lymph nodes without evidence of metastasis (20). These phenomena may represent microenvironmental changes induced by the primary tumor prior to metastasis and may be a future site for a secondary tumor (21). However, we deduce that these phenomena may reflect a host antitumor reaction and prohibit metastatic tumor cell growth.

The siRNAs used in this study were chemically modified to allow broad biodistribution and effective cell entry (22). In addition, in vivo gene electrotransfer was applied as a means of delivering siRNAs to the tumor cells. This technique offers some attractive advantages, such as high efficiency of DNA transfer in vivo, ease of manipulation and high gene accumulation through repeated transfections, providing an efficient method of delivering genes into tumors (23). Our results demonstrated that decreasing VEGF gene expression in bladder cancer cell tumors by this means suppressed tumor growth and metastasis. Thus, siRNA-mediated inhibition of 
VEGF-A, VEGF-C or VEGFR-3 expression may be an option for arresting tumor growth and metastasis and improving disease-free survival rates of patients with bladder cancer.

\section{Acknowledgements}

This study was supported by grants from the National Basic Research Program of China (973 project, 2004CB518804), the National Natural Science Foundation of China (30325043), the Project for Talent Accomplishment of the Health Bureau of Shanghai, China (99BR006), and the Rising-Star Program of Shanghai, China (04QMX1417).

\section{References}

1. Stacher SA, Achen MG, Jussila L, Baldwin ME and Alitalo K Lymphangiogenesis and cancer metastasis. Nat Rev Cancer 2 573-583, 2002.

2. Carmeliet $\mathrm{P}$ and Jain RK: Angiogenesis in cancer and other diseases. Nature 407: 249-257, 2000.

3. Ferrara N, Gerber HP and LeCouter J: The biology of VEGF and its receptors. Nat Med 9: 669-676, 2003.

4. Saban MR, Towner R, Smith N, et al: Lymphatic vessel density and function in experimental bladder cancer. BMC Cancer 7 : 219, 2007.

5. Mylona E, Magkou C, Gorantonakis G, et al: Evaluation of the vascular endothelial growth factor (VEGF)-C role in urothelial carcinomas of the bladder. Anticancer Res 26: 3567-3571, 2006.

6. Fire A, Xu S, Montgomery MK, Kostas SA, Driver SE and Mello CC: Potent and specific genetic interference by doublestranded RNA in Caenorhabditis elegans. Nature 391: 806-811, 1998.

7. Ferrara N: Molecular and biological properties of vascular endothelial growth factor. J Mol Med 77: 527-543, 1999.

8. Wang F, Wu JH, Tian YH, et al: Role of VEGF in the growth and metastasis of a murine bladder carcinoma. Chin Sci Bull 48: 2404-2410, 2003.

9. Joukov V, Pajusola K, Kaipainen A, et al: A novel vascular endothelial growth factor. VEGF-C, is a ligand for the Flt-4 (VEGFR-3) and KDR (VEGFR-2) receptor tyrosine kinases. EMBO J 15: 290-298, 1996.

10. Oh SJ, Jeltsch MM, Birkenhäger R, et al: VEGF and VEGF-C: specific induction of angiogenesis and lymphangiogenesis in the differentiated avian chorioallantoic membrane. Dev Biol 188: 96-109, 1997
11. Zu X, Tang Z, Li Y, Gao N, Ding J and Qi L: Vascular endothelial growth factor-C expression in bladder transitional cell cancer and its relationship to lymph node metastasis. BJU Int 98: 1090-1093, 2006

12. Hammond SM, Caudy AA and Hannon GJ: Post-transcriptional gene silencing by double-stranded RNA. Nat Rev Genet 2: 110-119, 2001.

13. Devi GR: siRNA-based approaches in cancer therapy. Cancer Gene Ther 13: 819-829, 2006.

14. Karagiannis TC and El-Osta A: RNA interference and potential therapeutic applications of short interfering RNAs. Cancer Gene Ther 12: 787-795, 2005.

15. Elbashir SM, Harborth J, Lendeckel W, Yalcin A, Weber K and Tuschl T: Duplexes of 21-nucleotide RNAs mediate RNA interference in cultured mammalian cells. Nature 411: 494-498, 2001.

16. Wang F, Tian YH, Chen XF and Huang Q: Microarray analysis the role of VEGF signaling pathway for the growth and metastasis of routine bladder earcinonla. Tumor 27: 847-885, 2007.

17. Sun P, Gao J, Liu YL, Wei LW, Wu LP and Liu ZY: RNA interference (RNAi)-mediated vascular endothelial growth factor-C (VEGF-C) reduction interferes with lymphangiogenesis and enhances epirubicin sensitivity of breast cancer cells. Mol Cell Biochem 308: 161-168, 2008

18. Jia RB, Zhang P, Zhou YX, et al: VEGF-targeted RNA interference suppresses angiogenesis and tumor growth of retinoblastoma. Ophthalmic Res 39: 108-115, 2007.

19. Guan H, Zhou Z, Wang H, Jia SF, Liu W and Kleinerman ES: A small interfering RNA targeting vascular endothelial growth factor inhibits Ewing's sarcoma growth in a xenograft mouse model. Clin Cancer Res 11: 2662-2669, 2005.

20. Ioachim HLRH: Tumor-Reactive Lymphadenopathy. 3rd edition. Lippincott Williams and Wilkins, Philadelphia, pp254-258, 2002.

21. Qian CN, Berghuis B, Tsarfaty G, et al: Preparing the 'soil': the primary tumor induces vasculature reorganization in the sentinel lymph node before the arrival of metastatic cancer cells. Cancer Res 66: 10365-10376, 2006.

22. Manoharan M: RNA interference and chemically modified small interfering RNAs. Curr Opin Chem Biol 8: 570-579, 2004.

23. Wang F, Chen XF, Tian YH, Wu JH, Li L, Li CY and Huang Q: Target gene transfer mediated by electroporation for cancer therapy in vivo. Prog Biochem Biophys 29: 734-740, 2002. 